\title{
Mitigating the Impact of Mobility on H.264 Real-Time Video Streams using Multiple Paths
}

\author{
Carlos T. Calafate, Manuel P. Malumbres, and Pietro Manzoni
}

\begin{abstract}
One of the main problems associated with MANETs is that mobility and the associated route discovery and maintenance procedures of reactive routing protocols cause severe interruptions on real-time video streams. Some of these interruptions are too large to be concealed using any sort of video technology, resulting in communications breaks unpleasant for the final end user. We present a solution for enhanced video transmission that increases route stability by using an improved route discovery process based on the DSR routing protocol, along with traffic splitting algorithms and a preventive route discovery mechanism. We also present some video adaptative mechanisms that improve the overall performance of multipath routing in terms of video data replication and video packet splitting strategies.

Combining our proposals, we achieve up to $97 \%$ less interruptions on communication with high mobility and over $1.2 \mathrm{~dB}$ of improvements in terms of video distortion.
\end{abstract}

Index Terms: H.264, MANETs, multipath routing, video streaming.

\section{INTRODUCTION}

Mobile ad hoc networks, also known as MANETs, are composed by a set of independent mobile nodes which "cooperate" without any type of infrastructure. The low cost and ease of deployment of this kind of networks make them extremely attractive for applications ranging from military and disaster relief situations to small home environments. These networks have been under intensive research in the last few years and more and more applications are being found for MANETs.

The JVT H.264 / MPEG-4 part 10 standard [1], jointly developed by the ITU-T VCEG and ISO MPEG-4 groups, is a new video technology able to achieve very good video quality levels at low bit-rates as well as enhanced error resilience. Such properties make it adequate for MANET environments, which are bandwidth constrained and error-prone networks.

Current solutions in terms of routing protocols, access to the wireless medium, etc. are acceptable for best-effort traffic. However, the current performance of MANETs can hardly be accepted for real-time multimedia communication.

In [2], we found that even when a video flow does not have to face competition with other flows, and when the routing protocol operates in optimal conditions, video performance is still penalized by mobility.

\section{Manuscript received July 30, 2004}

The authors are with the Department of Computer Engineering (DISCA), at the Polytechnic University of Valencia (UPV), Spain, email: \{calafate, mperez, pmanzoni\}@disca.upv.es.

This work was supported by the Ministerio de Ciencia y Tecnologia under grant TIC/2003/00339 and the Junta de Comunidades de Castilla La-Mancha under grant $\mathrm{PBC} / 03 / 001$.
In this work, we show that there is an intimate relationship between video gaps and route disruptions. To alleviate this problem, we propose a technique to reduce the number and size of video gaps by enhancing DSR [3] route discovery procedure. We then describe a traffic splitting approach which uses a route selection mechanism that optimizes the use of multiple disjoint routes. The use of disjoint routes reduces video gaps occurrence generated by node mobility, thus improving the quality of the received video. Finally, in order to prevent the occurrence of route losses we provide a preventive route discovery mechanism activated when a video flow does not have at least two disjoint routes available.

To further improve the results, we make an analysis of the optimal tuning of the H.264 codec focusing on optimal integration with the packet splitting mechanism in terms of packetization, frame types, and packet replication procedures. Relatively to the replication procedures, we analyze the improvements achieved by introducing video awareness in the replication process.

To measure the effect of video gaps over the final video quality delivered to the user, we propose a metric called "video annoyance" $(V A)$. This metric helps in evaluating the behavior of the proposed route discovery and traffic splitting mechanisms.

Concerning the structure of this paper, in Section II, we refer some related works. In Section III, we make a brief introduction to the DSR protocol and describe the different route discovery techniques proposed. Section IV presents the effects of applying enhanced route discovery procedures over the delivered video quality, performing a detailed study of the video loss pattern. In Section V, we present a multipath routing algorithm that introduces traffic splitting as a mechanism to improve final video quality when node mobility is significant. In Section VI, we perform a global evaluation of all mechanisms presented in this paper. Finally, in Section VII, we make our concluding remarks.

\section{RELATED WORK}

The use of multiple routes in MANETs has recently become a promising solution for multimedia data transmission.

Wang et al. [4] introduced a probing technique to assess the quality of available routes, so that the traffic is forwarded based on the delay of each route. Their objective was also to achieve load distribution as well as improved throughput, end-to-end delay, and queue utilization.

Nasipuri et al. [5] proposed a strategy for quick route recovery through packet re-direction in intermediate nodes to reduce the frequency of query floods. Their solution aims at reducing the number of lost route messages as well as performing fewer route discoveries. However, the source is unaware of any extra 
routes, which means that their solution does not aid in the task of splitting traffic through disjoint routes.

$\mathrm{Wu}$ [6] proposes a more selective route discovery procedure than DSR to increase the degree of disjointness of routes found without introducing extra overhead. It allows the source to find a maximum of only two paths (node disjoint paths) per destination.

In [7], Lee and Gerla show that the paths found by DSR's route discovery mechanism are mainly overlapped, and so they propose an extension to find more paths.

In [8], the AODV protocol has been extended in order to provide multi-path capabilities, though no new route discovery mechanism was proposed. Both node disjoint and link disjoint approaches are presented. In their work, there is no traffic splitting.

Finally, in [9] the authors use multiple node disjoint routes as a basis for reliable routing through the deployment of reliable nodes.

In this paper, we will show how the availability of extra routes along with new algorithms to optimize path disjointness, reduces the chances of losing all routes to a destination. Such methods prove to be very effective in reducing video gaps.

\section{ROUTE DISCOVERY EXTENSIONS TO DSR}

The dynamic source routing (DSR) protocol [3] is a high performance protocol for MANETs. Its route discovery process is on-demand, which means that routes are only built when needed; route maintenance also depends on the existence of traffic. Therefore, when there is no traffic flowing in the network, the routing traffic is effectively reduced to zero.

When initiating a route discovery, the source broadcasts a route request (RREQ) packet, which is successively broadcasted by other nodes until the destination is reached. When relying on IEEE 802.11 for transmission, packet broadcasting suffers from noise and collisions; this means that there is no assurance that the best route will be discovered, or that a request will arrive to the destination at all. Route replies, on the other hand, are unicast packets and, when using IEEE 802.11, this means that they are acknowledged so communication is more reliable.

DSR is a protocol that, by default, finds only a small number of routes. By extending the route discovery mechanism in DSR we are able to increase the average number of routes found per node. This extra information alone offers to each node extra possibilities when a route is lost, requiring on average fewer route discovery processes. As an enhancement, nodes can use the extra information for other useful purposes, like packet splitting or replication over disjoint routes, route congestion analysis, etc. Although the enhancements proposed have a bandwidth cost on route discovery actions, we believe that this cost is acceptable, especially taking into consideration the trend towards increased bandwidth in recent standards.

With our route discovery proposal (see [10]), we decrease the routing overhead when compared to the Lee and Gerla's route discovery technique. Their proposal consists of altering the route discovery process so that during the "RREQ" propagation phase packets with the same route request ID can be forwarded if they arrive "through a different incoming link than the link

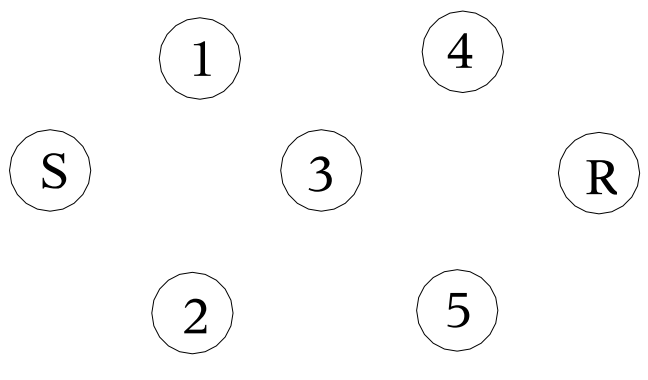

Fig. 1. Example scenario.

from which the first RREQ is received, and whose hop count is not larger than the first RREQ".

From now on, we shall refer to our solution as "super restrictive" mode (SR). In SR mode, we add a list (SRlist), to the already existing route discovery table structure in all nodes. This list is used to store the intermediate hops that forwarded the route request. The cost in terms of memory is very smallonly 4 bytes per intermediate hop per source for each list entry. The main enhancement of the SR mode is that it discontinues the propagation of a route request if some of the previous hops (except the source) are the same. With this method we assure that the discovered paths are node disjoint, increasing therefore the usefulness of the routes found.

To evidence the difference between node and link disjoint paths, we take as an example the scenario of Fig. 1 where source $\mathrm{S}$ communicates with destination $\mathrm{R}$.

In this case, we can find paths which are both node and link disjoint such as $\{\mathrm{S}-1-4-\mathrm{R}\}$ and $\{\mathrm{S}-2-5-\mathrm{R}\}$, and we can also find paths that are link disjoint only such as $\{\mathrm{S}-1-3-4-\mathrm{R}\}$ and $\{\mathrm{S}-2-3-$ $5-\mathrm{R}\}$. In the latter case, node 3 is a common intermediate node, which explains why it is not node disjoint. We will further detail the usefulness of link and node disjoint paths in Section V-A.

The SRlist, used to assure that the node disjointness condition on RREQ propagation is achieved, can have its size controlled and limited. When a route request arrives and the list is already full, it is not propagated. This means that only a pre-defined number of route requests are forwarded. If the size of the SRlist is very high we obtain the basic SR mode; if it is equal to one the behavior is similar to the DSR's propagation mode. The size of the SRlist is a new parameter and it will be referred to as PNC (previous node count).

The SR solution restricts the route request forwarding process to route sizes not superior to the first one arriving. To increase the flexibility of the approach we accept routes with an extra size up to a certain value. We call this parameter flexibility. In [10], we found that only small values for this parameter are useful in terms of route size and stability.

In the following sections, we test three different Flexibility / $P N C$ pairs. In mode $1($ Flex $=0, P N C=2)$, the propagation using the SR technique is restricted to the maximum, so that only one extra route per node is allowed relative to the default DSR behavior. Modes 2 and 3 maintain one of the parameters of mode 1 , but in mode $2($ Flex $=2, P N C=2)$ we increase flexibility, and in mode 3 (Flex $=0, P N C=4)$ we increase the number of RREQs propagated per node. In all modes the use of cache on route propagation is turned off, maintaining the rest of DSR's behaviors unchanged. 


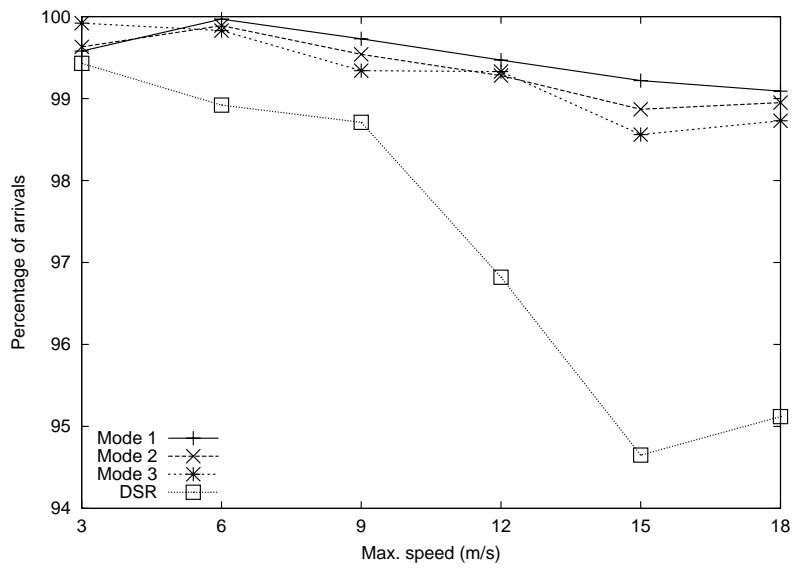

(a)

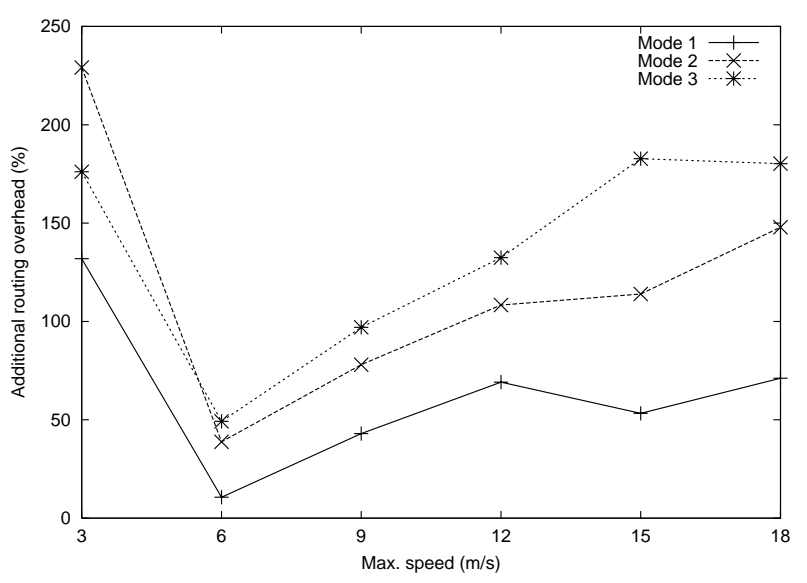

(b)

Fig. 2. H.264 video flow performance versus node speed: (a) Percentage of arrivals, (b) routing overhead.

\section{EFFECTS OF ROUTE STABILITY ON REAL-TIME VIDEO STREAMS}

In this section, we put in evidence the presence of gaps in video streams, evaluating the performance of DSR and the three different SR modes when facing this problem. We show that video gaps are intimately related to route discovery procedures, and why the SR modes presented in the previous section can considerably alleviate the video gap problem.

The evaluations are done using the ns-2 simulator [11]. Each obtained value is an average over 5 simulation runs. Concerning node movement, it was generated using the random waypoint mobility model. A filter was applied to accept only scenarios without network partitioning (i.e., with no unreachable destinations) in order to obtain a connected graph.

We first evaluate a $1000 \times 1000$ meters squared scenario with 80 nodes. The traffic load consists of a single H.264 video stream obtained from the well known Foreman sequence at 10 frames-per-second. Each video frame is split into 7 RTP packets, resulting in a target bit-rate of $186 \mathrm{kbit} / \mathrm{s}$. Our purpose is to observe the performance of the different routing protocols independently from other traffic flows.

In Fig. 2, we observe how modes 1 to 3 always perform slightly better that the original DSR implementation in terms of packet arrivals. The best performing mode is SR mode 1, where the improvements over DSR reach $4.5 \%$ in packet arrivals. Concerning routing overhead, it generates fewer routing control packets than the remaining two, with a small relative increase compared to DSR.

\section{A. Loss Pattern Analysis}

The H.264 standard offers a wide range of tools to reduce the effects of degradation in the presence of losses. Different types of intra macroblock updating strategies and error concealment tools are available, which aims at estimating parts of frames which are not received. Thus, observing the results of Fig. 2 and taking into account these facts we could conclude that the difference in terms of PSNR (peak signal-to-noise ratio) between receiving $99 \%$ or $95 \%$ of the packets would be slight.

However, packets dropped in bursts long enough to cause video gaps affect the H.264 video decoder in a drastically different manner. When no information relative to consecutive frames arrives to the decoder, it will freeze the last decoded frame until communication is resumed. When communication is resumed the decoder's effort is also increased since it must resynchronize and recover from losses as quickly as possible [12]. We therefore argue that the PSNR is not a representative factor and we propose a new metric which we called VA (video annoyance) parameter defined as

$$
V A=\frac{\sum_{i=0}^{N}\left(G_{i}\right)^{2}}{N F^{2}}, 0 \leq V A \leq 1,
$$

where $N F$ is the total number of frames in the sequence, $N$ is the total number of independent video gaps occurring in a video sequence, and $G_{i}$ is the size of the $i$-th video gap. We define a video gap $G_{i}=\frac{B_{i}}{P P F}$ as the number of video frames lost sequentially; $B_{i}$ is the number of consecutive packets lost for gap $i\left(B_{i} \geq 2\right)$ and $P P F$ is the number of packets per frame.

This number does not need to be an integer since for example $4 \frac{1}{2}$ frames can be lost in a single burst being communication resumed with information relative to some position inside a frame. What must be defined, though, is the minimum number of consecutive lost packets to create a video gap. In this work we set that threshold to one entire frame, that is, 7 packets $\left(B_{i} \geq 7\right)$.

Concerning $V A$ limits, when $V A=0$ there are no video frame gaps; when $V A=1$ the entire sequence is lost. The quadratic relation takes into account the fact that many distributed 1-frame gaps are almost imperceptible to the viewer, while a single 50 frames gap ( 5 seconds interruption at $10 \mathrm{~Hz}$ ) is quite disturbing for the user.

Analyzing a typical packet drop pattern on a simulation using DSR and a single H.264 video flow, we can observe (see Fig. 3) that some of the packet loss events are bursty. This causes the video flow to be stopped so that several entire frames are lost.

Table 1 presents a comparison between the different routing protocols concerning the $V A$ parameter. As it can be seen, the $V A$ associated with modes 1 to 3 is only a small fraction (1$2 \%$ ) of the $V A$ achieved with the original DSR implementation. 
Table 1. Video annoyance statistics.

\begin{tabular}{|c|c|c|}
\hline Protocol & $V A\left(10^{-6}\right)$ & $V A \%$ towards DSR \\
\hline \hline DSR & 38.1 & - \\
\hline Mode 1 & 0.502 & 1.3169 \\
\hline Mode 2 & 0.708 & 1.8575 \\
\hline Mode 3 & 0.768 & 2.0167 \\
\hline
\end{tabular}

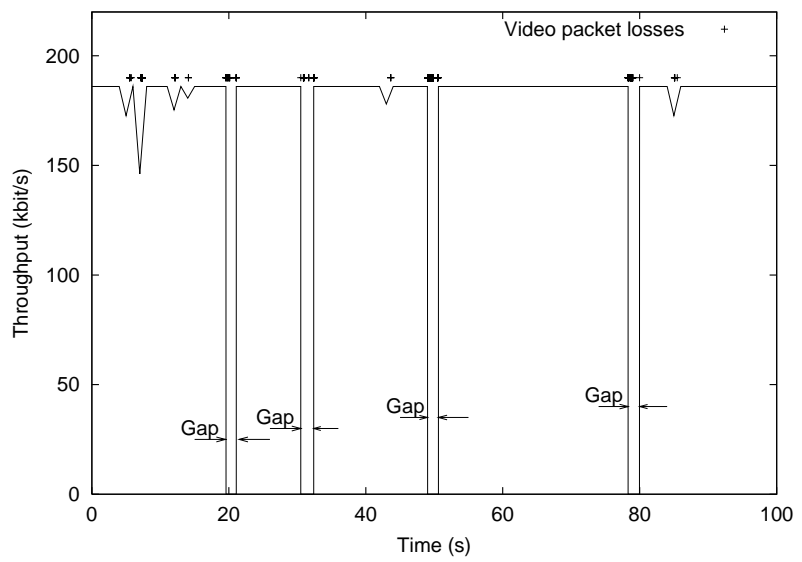

Fig. 3. Video throughput variation.

This result alone proves how the different SR modes improve the video experience in terms of video disruptions.

To further analyze and validate the improvements shown with the $V A$ parameter, we study (see Fig. 4) the video gap histogram for all protocols at maximum node speed. DSR performs much worse for all gap intervals with respect to the SR modes, being mode 1 the one that achieves the best results.

Another important factor shown in Fig. 4 is that SR modes 1 to 3 present gap sizes of no more than 20 frames, contrarily to DSR. In fact, DSR is prone to lose as much as 217 consecutive frames (more than 20 seconds of interruption), while for mode 1 the maximum gap size experienced is of only 13 frames $(1.3$ seconds interruption).

\section{B. Gap Causes and Solutions}

In the scenarios where congestion is not a problem like the ones we used, packet losses are intimately associated with link breaks and subsequent route failures.

However, DSR uses link layer information to detect broken links, so the interval between the detection of a broken link and the reception of associated notification by the source is, in terms of video streaming, not excessively long. In fact, we find that when a route breaks the number of packets lost can be estimated by

$$
N=2 S_{\text {rate }} T_{R E R R},
$$

where $S_{\text {rate }}$ is the source's packet generation rate in packets per second and $T_{R E R R}$ is the time that the "route error" packet takes to arrive at the source. This phenomena can sometimes be alleviated by DSR's packet salvaging mechanism.

We therefore conclude that the cause for such long video gaps is related to route discovery procedures, and not just route failures. Fig. 5(a) aids at proving this statement. As it can be seen,
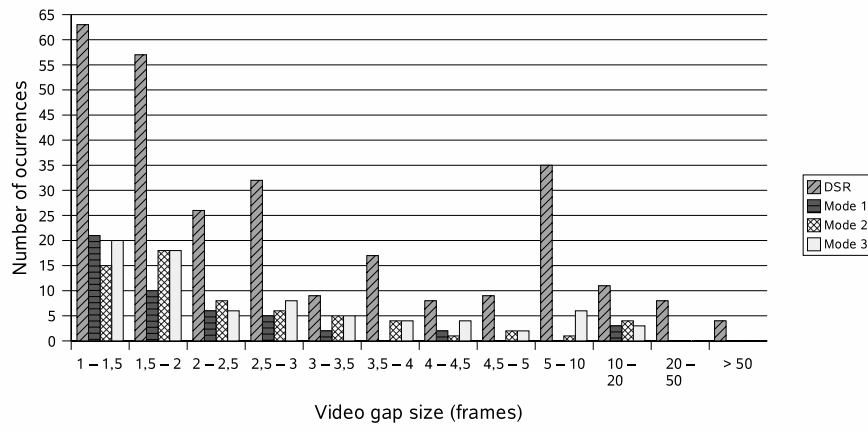

Fig. 4. Video frame gaps histogram at a maximum node speed of 18 $\mathrm{m} / \mathrm{s}$.

only RREQ procedures force the number of consecutive packets dropped to be above the gap threshold, while route failures (RERR) events are usually not associated with video gaps at all. We also verified that in this scenario all video gaps are associated with a route request (RREQ) event since there is no competing traffic. Relative to the gap threshold, it is equal to 7 packets (one frame) as defined previously.

Proven this direct relationship between video gaps and route request events, we studied the behavior of the different SR modes relative to DSR in terms of RREQs in order to provide a clear explanation of the results of Table 1 and Fig. 4. We calculated the number of route requests generated by the video source at different speeds achieving the results presented on the bottom side Fig. 5. Results are relative to those found for the DSR protocol.

From these results, we can see that modes 1 to 3 present less video gaps for all speeds as expected due to the higher number of routes found. The relationship between the number of RREQs and video gaps also explains the improvements in terms of $V A$. The mode with best overall performance (mode 1 ) shows an average reduction of $68 \%$ on the number of RREQs generated in relation to DSR, and so we will use it as a basis for the improvements performed in the following sections.

\section{MULTIPATH ROUTING}

In the previous section, we have shown that video streams flowing in MANETs are prone to be degraded through packet loss bursts which cause gaps in communication. We presented some techniques to measure those gaps such as the $V A$ parameter and the video gaps histogram and proved that using any of the SR modes proposed we can alleviate the video quality degradation introduced by long packet loss bursts. We also showed that video gaps are intimately related to route discovery processes.

In this section, we analyze how the use of simultaneous paths on data transmission effectively reduces the down-time of multimedia flows making the communication experience smoother and more pleasant to the user. The use of multipath routing can be extended to any type of data. In this proposal, we concentrate on multimedia streams alone.

We consider that out-of-order delivery provoked by multipath routing is not a primary issue. In fact, in the process of packetization of the raw video stream into RTP packets-functionality 


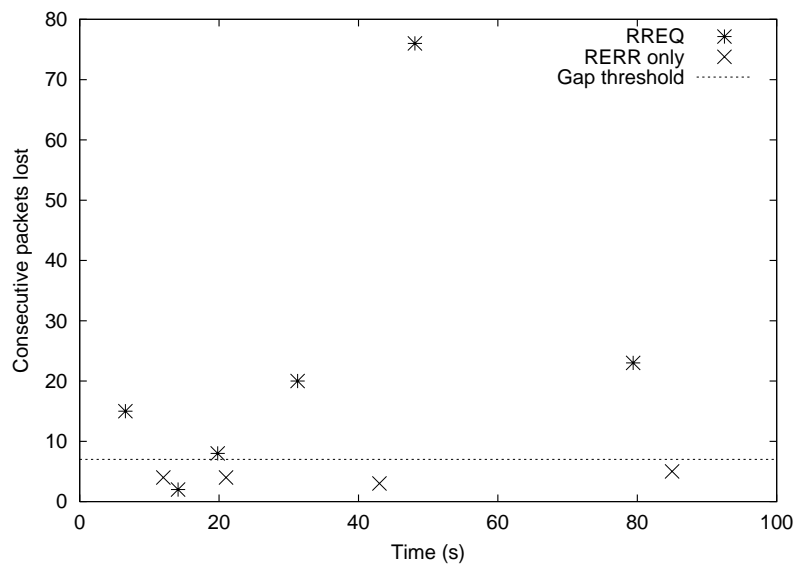

(a)

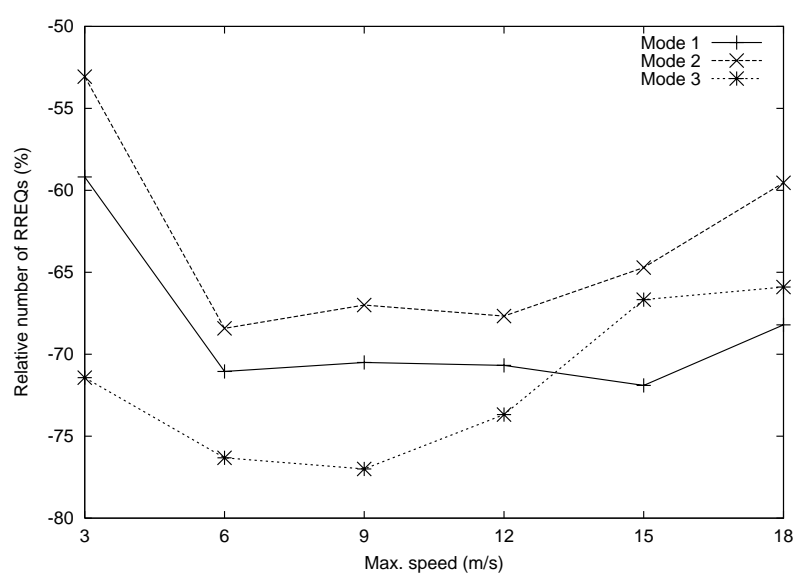

(b)

Fig. 5. Performance comparison: (a) Relationship between RREQs, RERRs, and video gaps, (b) relative number of RREQs generated.

integrated in the H.264 codec-sequence numbers are included in RTP header, which allows the receiver to reconstruct the sending order. Also, the video decoder can use sequence numbers to determine the proper location of a packet without necessarily decoding packets in sequence.

\section{A. Traffic Splitting Strategies}

Traffic splitting in the context of multipath routing refers to the technique of distributing the packets of a certain stream through different paths. Concerning the paths themselves, we can talk about their degree of disjointness and also make a distinction between link disjoint and node disjoint paths [8]. Node disjoint paths are those where none of the intermediate nodes are in common. Link disjoint paths are those where all links differ (see Section III).

The optimal strategy in terms of traffic splitting would be using the shortest disjoint paths. In general, node disjoint paths are preferable since these achieve the best use in terms of both bandwidth and node resources. There are some cases where no node disjoint paths are available and, therefore, link disjoint paths are recommendable. In fact, the link disjointness condition is

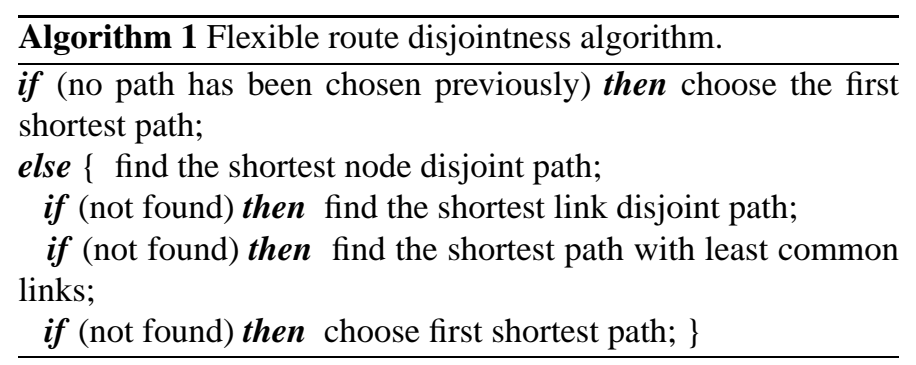

enough to reduce the effect of mobility on ad hoc networks. The algorithm we propose for maximum disjointness is Algorithm 1.

With Algorithm 1 we are able to completely detach the route discovery and maintenance process from the packet splitting process. This is true because the algorithm is able to dynamically adapt itself to new discovered routes, and so does not impose any demand on the route discovery process itself nor on the destination. From now on, we shall refer to Disjoint solution as the one which makes use of this algorithm for traffic splitting and uses SR mode 1 for the propagation of route requests.

The action of finding the disjointness of one route is always done relatively to the previously used route. This technique allows to easily adapt to extra routes found through the forwarding or interception of packets, as well as to routes which are no longer valid. To provide a good basis of comparison we define a metric that clearly evidences the exact gains in terms of path dispersion, that is, an average degree of path disjointness. So, the dispersion between two consecutive paths for a single stream would be

$$
\text { Dispersion }=1-\frac{C L}{N L},
$$

where $C L$ is the number of common links relative to the previous path and $N L$ is the number of links of the current path.

We aim at dispersion values near to 1 , which is the optimal solution; dispersion values near 0 reflect a bad traffic dispersion policy.

Though this algorithm aims at finding the best choices in each situation, it could be considered computationally expensive for small embedded systems. Therefore, we also propose a much simpler solution which consists of randomly choosing routes which are not larger than a certain size $(s)$ relative to the first one. This alternative solution, referred as $R_{s}$, aims at providing a comparison basis of the goodness of the Disjoint solution.

Using the same simulation setup used in Section IV, we evaluated the Disjoint algorithm comparing it with the $R_{s}$ solution.

Table 2 shows the average results by setting $s=2$ and varying the maximum allowed node speed from 3 to $18 \mathrm{~m} / \mathrm{s}$. Values presented are average values. As it can be seen, the maximally disjoint solution always achieves the best results.

In terms of end-to-end delay, the Disjoint solution always performs quite better than the $R_{2}$ one, which means that the paths used are shorter. In what refers to the routing overhead again the Disjoint solution performs much better.

If we observe the results concerning the dispersion achieved with both methods, we verify that the Disjoint solution presents dispersion values that more than double the ones from solution $R_{2}$. We also verify that the dispersion value almost does not vary with speed. 
Table 2. Comparison of traffic splitting strategies.

\begin{tabular}{|c|c|c|c|c|}
\hline Mode & $\begin{array}{c}\text { Video } \\
\text { arrivals (\%) }\end{array}$ & $\begin{array}{c}\text { Routing } \\
\text { overhead }\end{array}$ & $\begin{array}{c}\text { End-to-end } \\
\text { delay (ms) }\end{array}$ & Dispersion \\
\hline \hline Disjoint & 99.70 & 6759 & 39.54 & 0.71 \\
\hline$R_{2}$ & 97.60 & 11346 & 51.19 & 0.32 \\
\hline
\end{tabular}

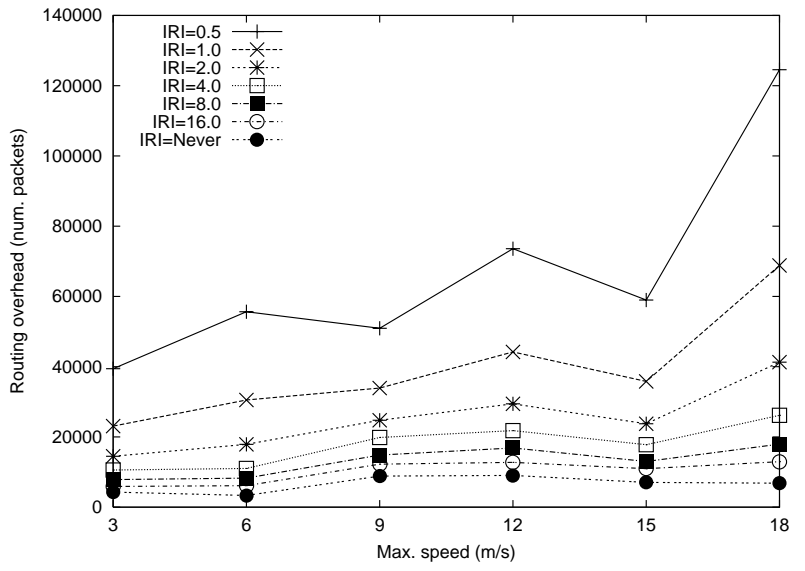

Fig. 6. Effect of inter-request interval on routing overhead.

This analysis allows us to conclude that the results achieved fully justify the extra computational complexity required by the Disjoint solution, being the $R_{2}$ a possible solution for environments with few resources, though the performance suffers some deterioration. From now on we will always use the Disjoint solution when performing traffic splitting.

\section{B. Preventive Route Discovery}

In order to improve the traffic splitting Disjoint strategy presented in the previous section, we propose a mechanism to perform preventive route discovery processes. Its objective is to minimize the video gap effects on the video quality delivered to the user.

By default, the DSR protocol initiates a route discovery process when it has no available routes to the destination. So, until a new valid route is discovered, our video flow transmission will lose a burst of packets, producing a video gap. We propose to perform preventive route discovery processes in order to avoid that situation. So, we think that it is reasonable to look for new routes when there are no disjoint paths available.

We also have to consider the possibility that, after completing a preventive route discovery cycle, no disjoint routes are found. In this case, we have to start another route discovery process to avoid video flow stall if the current route is lost. The rate at which we generate preventive route discovery processes must be evaluated in order to be useful and not to overload the network. By varying the preventive route discovery period among values: $0.5,1,2,4,8,16$, and never (default), we calculated the routing overhead differences among the various inter-request values (see Fig. 6). Remind that when all routes to the destination are lost, a new route discovery process is started and the probability of producing a video gap is high.
As expected, the routing overhead is higher than the default solution in all cases. The lower the inter-request interval, the higher the routing overhead becomes. We consider that for values under 4 seconds the routing overhead becomes prohibitive. In terms of packets arrivals, around $99 \%$ of packets arrive for all solutions under test at all speeds.

Considering the $V A$ parameter, we achieve the best average results for an inter-request interval of 8 seconds (about 1/3 compared with the default solution), which also offers very good results in terms of packet arrivals and routing overhead.

\section{Enhancements Achieved using Multipath Routing}

In this section, we are going to perform a global evaluation of DSR, SR (mode 1), and Disjoint solutions, adjusting the best options found for them. The simulation setup is configured with a $1000 \times 1000$ meters squared scenario and 80 nodes. The mobility pattern is generated using the random waypoint mobility model bundled in ns- 2 and an extra script was created in order to accept only scenarios where node topology forms a connected graph.

Only one video flow conforms the injected traffic with the same characteristics described in Section IV. The Disjoint solution uses a multipath routing algorithm with a preventive route discovery mechanism set to minimum period of 8 seconds.

We can clearly see on the top side of Fig. 7 how DSR performs worse than the remaining modes for moderate/high speeds. The Disjoint mode is the best for all speeds, but the SR mode alone already provides very good enhancements.

In terms of routing overhead, we can see in Fig. 7 that the SR mode does not generate an excessive number of control packets. The Disjoint mode causes more routing overhead since this protocol is performing preventive route discoveries frequently. Also notice that the rate of growth between the three solutions is quite different. Comparing the routing overhead for the minimum and maximum speeds we can see that while DSR control packets have increased by a factor of 9, SR and Disjoint modes have increased by factors of 5 and 2, respectively. This shows the better adaptation and appropriateness of the last two to high mobility scenarios. We also show the routing overhead achieved with the Disjoint mode when turning off the preventive route discovery mechanism (No PRD in Fig. 7). We can see that the routing overhead is highly reduced when that mechanism is turned off, showing an overhead similar to the SR mode.

If we now focus on our main goal-reducing video streaming gaps-we find that there has been a gradual improvement as shown in Fig. 8. As it can be seen, the SR mode is able to significantly reduce the video gap occurrence. With the Disjoint mode the improvements are even higher, keeping the size of most video gaps below 1.5 frames. 


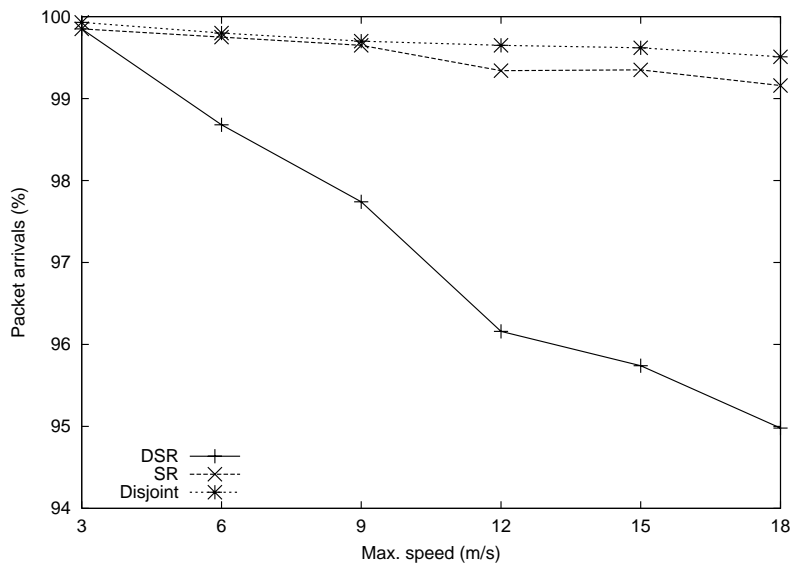

(a)

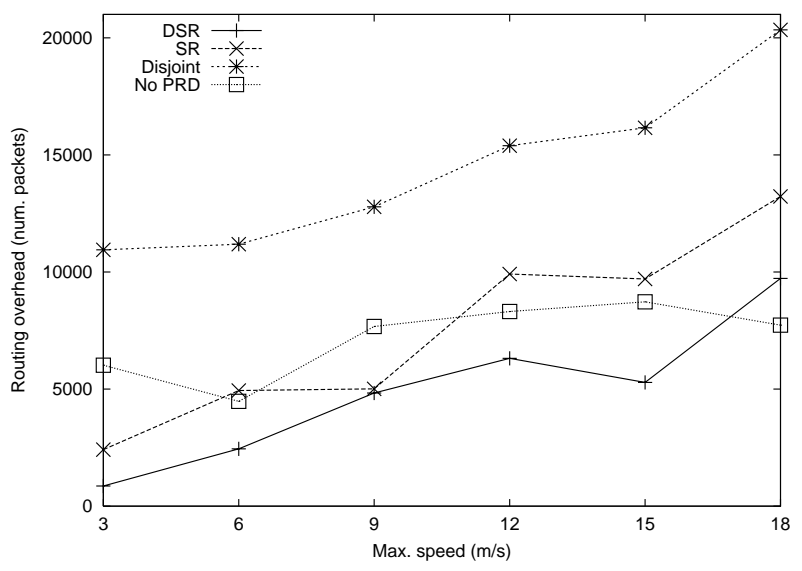

(b)

Fig. 7. Comparison of protocols in terms of (a) video packet arrivals and (b) routing overhead.

Table 3 shows the improvements in terms of global gap percentage and the $V A$ parameter. The reason why the difference in terms of $V A$ between SR and Disjoint modes is not greater is due to the fact that both approaches avoid large video gaps being large video gaps those that provoke significant differences in terms of $V A$ metric. For video gaps of less than 3 frames, though, the disjoint mode shows its effectiveness without doubt.

Finally, relative to the benefit of including or not the preventive route discovery mechanism, we achieve a slight increase in routing overhead and a reduction of $60 \%$ in terms of $V A$ and of $50 \%$ in gap percentage by turning it on. The main reasons for this difference are the few situations when preventive routing saves us of video gaps.

\section{VIDEO ENHANCEMENTS FOR MULTIPATH SCENARIOS}

The techniques presented in the previous sections focus on enhancing the routing protocol to provide a better video service. In this section, we present new enhancements to the Disjoint solution by focusing on an optimal adaptation of video flows. We start with a study on the impact of video-aware packet replica-
Table 3. $V A$ parameter comparison.

\begin{tabular}{|c|c|c|c|}
\hline Protocol version & DSR & SR & Disjoint \\
\hline Gap (\%) & 2.41 & 0.303 & 0.0776 \\
\hline Avg. $V A\left(10^{-7}\right)$ & 32.3 & 2.14 & 0.619 \\
\hline
\end{tabular}

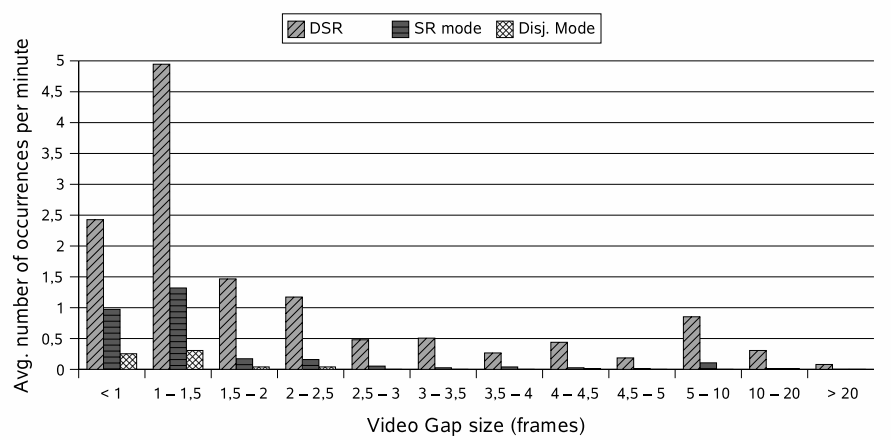

Fig. 8. Video gaps distributions for the 3 protocols under test at high mobility.

tion and proceed analyzing the need for good data distribution through different paths.

\section{A. Video-Aware Packet Replication}

Splitting traffic through two different routes towards a destination opens new possibilities for the enhancement of video streaming. A simple solution is to replicate all the information through both paths, so that if we have a packet loss probability $p$ on each path the receiver sees it as if losses were occurring with a probability of $p^{2}$. This way we achieve good improvements at twice the cost. If we analyze the video data organization we observe that not all the information is equally important. Intra coded frames, which are an essential source of reference for predicted frames, contain information which is more relevant than the rest. What we wish to achieve is, therefore, to improve the video distortion performance, but at the same time avoid an increase of $100 \%$ in the transmitted video data.

In our simulation analysis, we created an H.264 video of the Foreman sequence where $10 \%$ of the frames are intra-coded (I). The video metric we use to assess video quality is peak signalto-noise ratio (PSNR), which is also known as video distortion. From now on we will use both terms interchangeably.

The initial video distortion for our test sequence under no loss is of $33.51 \mathrm{~dB}$.

By simulating a statistically random loss pattern on both paths we analyzed the evolution of video distortion by varying the packet loss ratio from 1 to 20\%. The results presented in Fig. 9 are average values over 10 simulation runs.

The tag "None" indicates that no packet replication is being performed, which means that packets are just evenly split among the two paths. The "All" tag indicates a $100 \%$ packet replication. The remaining tags refer to the replication of $10 \%$ of the packets. In the experiment referred to as "Random" the replication process selects the $10 \%$ of packets in a random manner, while in the experiment tagged "I frames", the replication mechanism replicates only those packets related to I frames. 


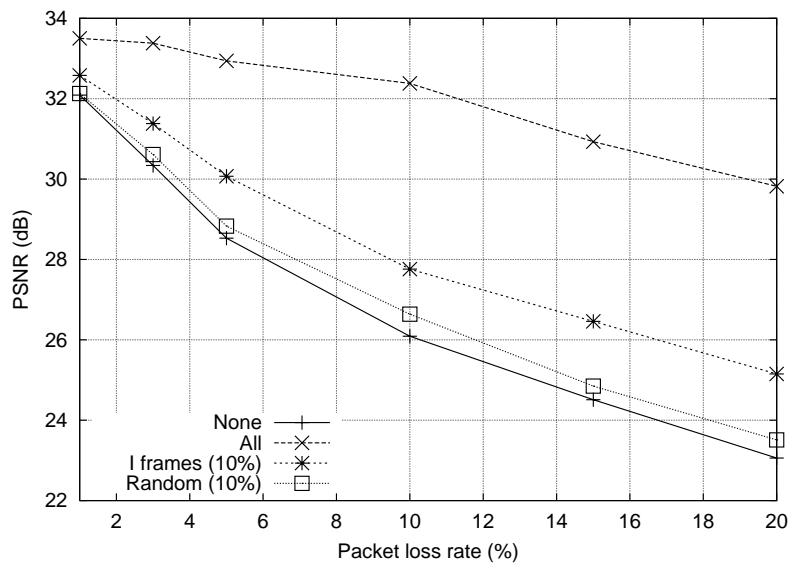

Fig. 9. Distortion values for different packet replication strategies.

From that figure, we find that by replicating $10 \%$ of the packets randomly, the distortion increases by $0.5 \mathrm{~dB}$ relative to noreplication, so it almost does not justify the $10 \%$ increase in bandwidth. We do achieve considerable gains by performing an intelligent packet replication, though still maintaining the same bandwidth increase. In fact, the introduction of video awareness in the packet replication process allows to achieve up to 2.14 additional dBs for packet loss rates of $20 \%$.

\section{B. Video Data Distribution in Multipath Scenarios}

We now focus on some aspects of video distribution in multipath scenarios. As exposed previously, packet splitting attenuates the effects of mobility by assuring that even when one path is lost, the other one remains available for data transmission. As soon as the loss is detected, the source stops sending packets through that invalid route. However, from the moment a broken link is detected to the moment the source receives the correspondent notification, a considerable amount of video packets could have been transmitted. Contrarily to random error scenarios, now the losses will affect well defined data-packets assigned to the invalid path. For example, if we split each frame in two packets and we route data through two disjoint paths, the loss of one path will always result in the loss of the upper or lower part of the frame, depending on the affected path. This situation will go on until the failure is detected.

To further examine this problem, we use the same video sequence as before (Foreman), and simulate the loss of one of the routes. Our evaluation envisaged scenarios where the source splits packets through 2 and 3 disjoint paths for comparison.

The results presented in Fig. 10 refer to the average distortion perceived in the first 10 affected frames. Also, when using 2 disjoint paths we average the result by losing one path or another; when using 3 paths we also average the 3 distinct possibilities.

We also consider that the affected frames may be intra coded (I) or predicted (P).

In Fig. 10, we can see that the number of packets per frame affects the sequence's distortion, with differences of up to $2 \mathrm{~dB}$. Here we have two distinct factors acting simultaneously: (1) A higher granularity in packetization will cause information loss to be spread in a more uniform manner throughout the frames. This

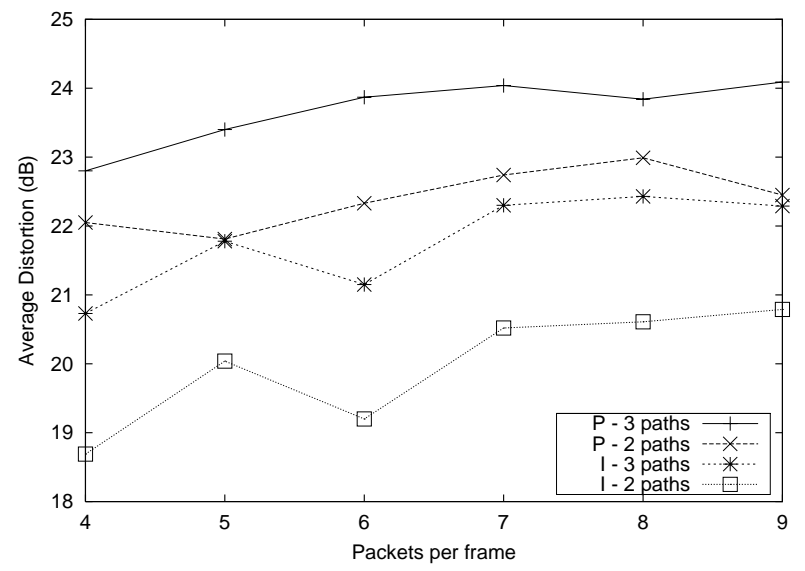

Fig. 10. Average distortion value for varying packetization granularity in the first 10 affected frames.

effect helps in increasing the quality, but has the drawback that it does also increase the network traffic. And (2), there is a relation between the number of packets per frame and the number of routes used. So, when the number of packets per frame is a multiple of the number of paths, the lost information will always refer to the same frame areas.

As a consequence of these interactions, we can see that in terms of video distortion using 7/8 packets per frame is the best solution for both predicted and intra-coded frames.

Another aspect shown in Fig. 10 and that should also be taken into consideration is that I frames are more severely affected from losses than $\mathrm{P}$ frames. However, once the route is established an intra-coded sequence will recover to maximum quality instantly, while sequences that rely only on prediction will recover slower. Therefore, a good balance between intra coded (I) and predicted $(\mathrm{P})$ frames on a sequence should be found in order to optimize simultaneously the usage of bandwidth and error propagation.

We tuned the sequence for a GOP size of 10 frames, that is, groups formed by an I frame followed by $9 \mathrm{P}$ frames.

\section{Video Enhancements Achieved}

To conclude our analysis we apply the different tools and methods developed until now to determine global improvements. The scenario is the same one used in Section IV, but now we add background traffic. $10 \%$ of the nodes are either sources or destinations. Each background source sends UDP traffic at a rate of four 512 byte packets per second.

Table 4 shows the results achieved with different protocol/replication combinations. In terms of packet arrivals for video data we see that the difference is minimum, except for DSR plus replication where the arrival percentage is slightly reduced. Concerning the use of the Disjoint mode with or without I frames replication, it causes a $2 \%$ increase in the background traffic, along with a considerable increase on the routing overhead as expected, though it can be reduced by about $50 \%$ if the preventive route discovery mechanism is turned off.

In terms of end-to-end delay, we found that except for very high packet arrival values, DSR alone performs better than the 
Table 4. Simulation results.

\begin{tabular}{|c|c|c|}
\hline Mode & $\begin{array}{c}\text { Video } \\
\text { arrivals (\%) }\end{array}$ & $\begin{array}{c}\text { Routing } \\
\text { overhead }\end{array}$ \\
\hline \hline DSR & 97.2 & 7011 \\
\hline DSR+Rep. & 95.9 & 6438 \\
\hline Disj. & 97.4 & 18380 \\
\hline Disj.+Rep. & 97.6 & 18953 \\
\hline
\end{tabular}

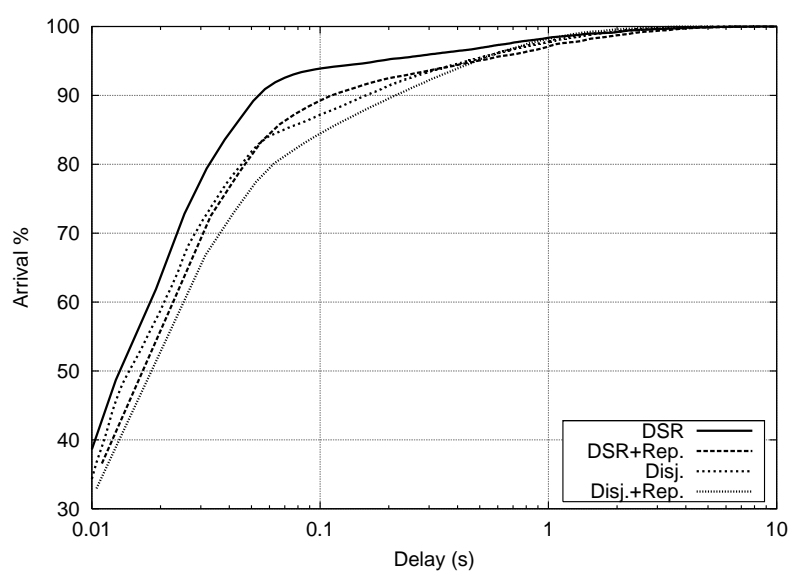

Fig. 11. Distribution function for video packet delays.

remaining modes as shown in Fig. 11. This result is expected since both packet splitting and packet replication mechanisms are prone to cause additional delays to a video stream. It is also interesting to notice that using multipath, $96 \%$ of the packets arrive faster without replication, but the remaining $4 \%$ arrive faster with replication on.

In order to assess the effect of the different strategies under study on the video stream, we evaluate the results in terms of average distortion for varying end-to-end delay thresholds. Such results are presented on Fig. 12. We should point out that the PSNR differences found are due to packet drops that are related to routing and delay thresholding, but are not related to congestion.

Contrarily to what would be expected by observing Fig. 11, DSR alone never performs better than is multipath counterpart. Concerning Disjoint mode plus replication, we also notice that it behaves better than DSR for end-to-end delay thresholds above $500 \mathrm{~ms}$.

In this scenario, it is not possible to observe the goodness of I frames replication obtained theoretically in Section VI-A since the main cause for packet losses is the loss of paths, which are not frequent, randomly distributed packet losses. It is clear, though, how the $10 \%$ increase in video traffic affects the overall performance, which leads us to think that this option shall be optimal only in situations where packet loss is more severe and more randomly distributed.

\section{CONCLUSIONS}

We presented several enhancements to the DSR protocol in order to provide a better support to real-time multimedia streaming, taking an H.264 video stream as a reference. We started by

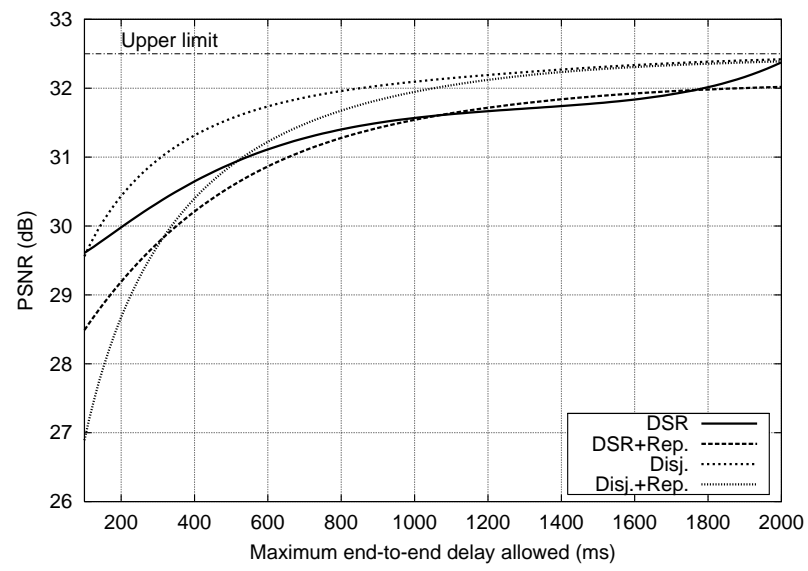

Fig. 12. PSNR variation for varying end-to-end delay thresholds.

presenting the SR mode, an extension to DSR's route discovery method which is part of a previous work. Afterwards, we evidenced the existence of video transmission gaps in wireless ad hoc networks. We studied their causes, as well as the effect of route stability on reducing this problem. Concerning video streams, we also proposed an alternative metric to PSNR, called video annoyance, in order to measure video gaps in a clear and straightforward manner.

We presented additional solutions to the video gap problem through packet splitting procedures and performing preventive route discoveries. The first of these mechanisms aims at reducing small but frequent video interruptions, while the second aims at eliminating very large video interruptions caused by the loss of all routes to destination. We achieved reductions in video gaps of up to $97 \%$.

After the multipath framework was set, we analyzed the interactions between the video codec and the packet splitting mechanism, tuning the former so as to obtain the best performance.

We concluded our work with an overall evaluation of the improvements achieved, showing that there are improvements in terms of video distortion values for different delay thresholds, where the mobility related gains can surpass $1.2 \mathrm{~dB}$.

\section{REFERENCES}

[1] Draft ITU-T Recommendation and Final Draft International Standard of Joint Video Specification (ITU-T Rec. H.264 | ISO/IEC 14496-10 AVC), Mar. 2003.

[2] C. T. Calafate, M. P. Malumbres, and P. Manzoni, "Performance of H.264 compressed video streams over $802.11 \mathrm{~b}$ based MANETs," in Proc. ICDCSW 2004, Hachioji - Tokyo, Japan, Mar. 2004.

[3] D. B. Johnson, D. A. Maltz, and Y.-C. Hu, "The dynamic source routing protocol," in Internet Draft, MANET Working Group, draft-ietf-manetdsr-10.txt, July 2004, work in progress.

[4] L. Wang et al., "Adaptive multipath source routing in ad hoc networks," in Proc. ICC 2001, vol. 3, 2001, pp. 867-871.

[5] A. Nasipuri, R. Castaneda, and S. R. Das, "Adaptive multipath source routing in ad hoc networks," Performance of multipath routing for on-demand protocols in mobile ad hoc networks, pp. 339-349, 2001.

[6] J. Wu, "An extended dynamic source routing scheme in ad hoc wireless networks," in Proc. 35th HICSS 2002, vol. 9, Big Island, Hawaii, Jan. 2002.

[7] S. Lee and M. Gerla, "Split multipath routing with maximally disjoint paths in ad hoc networks," in Proc. IEEE ICC 2001, 2001, pp. 3201-3205.

[8] M. Marina and S. Das, "On demand multipath distance vector routing in ad hoc networks," in Proc. IEEE ICNP 2001, 2001, pp. 14-23.

[9] Z. Ye, S. V. Krishnamurthy, and S. K. Tripathi, "A framework for reliable 
routing in mobile ad hoc networks," in Proc. IEEE INFOCOM 2003, San Francisco, USA, Apr. 2003.

[10] C. T. Calafate, M. P. Malumbres, and P. Manzoni, "A flexible and tunable route discovery mechanism for on-demand protocols," in Proc. 12-th Euromicro Conference on Parallel, Distributed and Network based Processing, La Coruña, Spain, Feb. 2004.

[11] K. Fall and K. Varadhan, "Ns notes and documents," in The VINT Project, UC Berkeley, LBL, USC/ISI, and Xerox PARC, Feb. 2000.

[12] C. T. Calafate and M. P. Malumbres "Testing the H.264 error-resilience on wireless ad-hoc networks," in 4th EURASIP Conference focused on Video / Image Processing and Multimedia Communications, Zagreb, Croatia, July 2003.

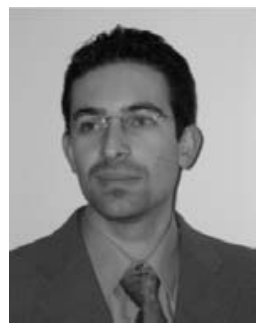

Carlos T. Calafate graduated with honors in Electrical and Computer Engineering at the University of Oporto (Portugal) in 2001. He received a Ph.D. grant from the Polytechnic University of Valencia (Spain) in 2002, and is since then a member of the Computer Networks Group (GRC) in the department of Computer Engineering. His research interests include mobile computing and networking, QoS on wireless networks, as well as video coding and streaming.

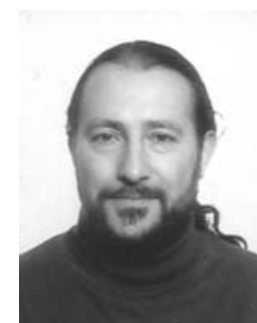

gies.
Manuel P. Malumbres received his B.S in Computer Science from the University of Oviedo (Spain) in 1986. In 1989 he joined to the Computer Engineering Department (DISCA) at Polytechnic University of Valencia (UPV), Spain, as an assistant professor. Then, he received the M.S. and Ph.D. degrees in Computer Science from UPV, at 1991 and 1996, respectively. In 2000 he became an associate professor and founded the Computer Networks Group (GRC). His research and teaching activities are related to multimedia networking, high-speed, and wireless network technolo-

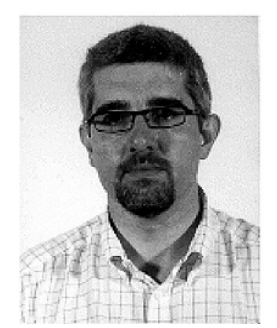

Pietro Manzoni is an associate professor of computer science at the Polytechnic University of Valencia (SPAIN). He received the M.S. degree in Computer Science from the "Università degli Studi" of Milan (ITALY) in 1989 and the Ph.D. in Computer Science from the Polytechnic University of Milan (ITALY) in 1995. His research activity is related to wireless networks protocol design, modeling, and implementation. 